\title{
Current Practices in the Assessment of Quality of Life in Individuals with Aphasia
}

\author{
K. Leigh Morrow-Odom'1, Dana C. Moser², Jean Neils-Strunjas ${ }^{3}$ \\ ${ }^{1}$ Department of Communication Sciences and Disorders, Western Carolina University Cullowhee, NC, USA; ${ }^{2}$ Department of Audiology and Speech \\ Pathology, University of Arkansas for Medical Sciences, University of Arkansas at Little Rock, AR, USA; ${ }^{3}$ Department of Communication Sciences \\ and Disorders, Western Kentucky University, Bowling Green, KY, USA
}

Purpose: The purpose of this study was to determine how speech-language pathologists (SLPS) in the United States are implementing recommendations regarding the assessment of quality of life (QoL) in persons with aphasia (PWA).

Methods: A brief, anonymous survey was made available online using a web-based survey platform. Questions addressed demographics, training, importance of QoL assessment, current patterns of clinical practice, and perception of barriers. Data reported included frequency counts and percentages.

Results: Sixty-two SLPs completed the survey in-part or in-full. The data suggest that the participants appreciate the importance of addressing QoL in PWA. Most participants indicated receiving instruction on QoL assessment in an academic setting, and about half reported completing continuing education on the topic. Interview and observation were most often used as the method of assessment, and few indicated using published measures. Information was elicited from a variety of sources, and QoL was most often assessed at the beginning and end of treatment, but seldom after discharge. QoL was less often assessed in those with severe and/or global aphasia. The most frequently reported barriers to assessing QoL were lack of training/knowledge and resources.

Conclusions: It is hoped that these findings will help facilitate improved clinical care for persons with aphasia by encouraging SLPs to utilize currently available tools for assessing QoL in PWA and work to develop other tools and strategies for conducting QoL assessments throughout the rehabilitation process, including into the chronic stage of recovery, in order to optimize recovery.

Keywords: Speech-language pathologist, Aphasia, Quality of life, Assessment, Current practice, Implementation, Instruction

\section{INTRODUCTION}

Aphasia is an acquired language disorder resulting from a brain injury that impairs one's ability to process language in receptive and expressive modalities. The severity and presentation of this impairment varies considerably depending on the location and extent of brain injury. As varied as the aphasia profiles are, so too are the effects of the language impairment on communication and quality of life (QoL). QoL is defined by the World Health Organization (WHO) Quality of Life Group as persons,' "perceptions of their position in life in the context of the culture and value systems where they

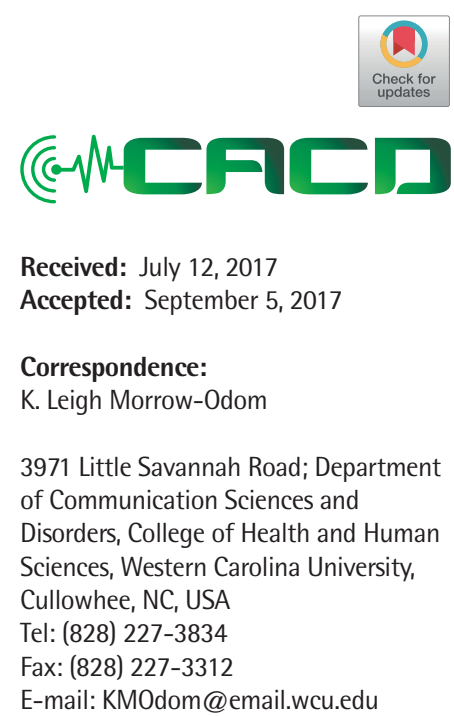

(C) 2017 The Korean Association of SpeechLanguage Pathologists

This is an Open Access article distributed under the terms of the Creative Commons Attribution Non-Commercial License (http:// creativecommons.org/licenses/by-nc/4.0/) which permits unrestricted non-commercial use, distribution, and reproduction in any medium, provided the original work is properly cited. 
live and in relation to their goals, expectations, standards and concerns" [1]. Research suggests that persons with aphasia (PWA) experience diminished QoL [2,3], and those with severe aphasia demonstrate more impaired QoL than those with mild-to-moderate aphasia [4]. Lam and Wodchis [5] found that aphasia is more detrimental to QoL than many other major health issues, including cancer and Alzheimer's disease. In PWA, QoL is often impacted by factors such as independence, social engagement, environmental supports, emotional distress, and concomitant health issues [6,7]. Aphasia rehabilitation may promote social participation, health and wellness to improve treatment outcomes $[8,9]$.

As it relates to aphasia, the WHO discusses QoL in terms of activity (i.e., communication) and its limitations (i.e., aphasia); social participation and its restrictions; communication facilitators and barriers; and personal factors. The American Speech-Language-Hearing Association (ASHA) Preferred Practice Guidelines [10] clearly state that the typical aphasia assessment should include examination of not only the language impairment itself but also the impact that it has on the person's life participation. In addition, the assessment should include identification of those factors hindering and facilitating successful communication and participation in life. Ross [11] states that SLPs have a multitude of options when selecting an appropriate tool for assessing QoL, with options ranging from generic to specific to comprehensive, and also variations regarding from whom responses can and should be elicited (e.g., patient, family, physician). Examples of QoL assessments appropriate for PWA include ASHA's Quality of Communication Life Scale (ASHA-QCL) [12] and the Stroke and Aphasia Quality of Life Scale-39 (SAQOL-39) [13]. The data obtained from these types of measures, combined with interview, observation and additional language testing, are critical in developing a treatment plan that addresses QoL needs.

Although QoL is emphasized in the current guidelines, little is known about how practicing SLPs attempt to address these QoL recommendations for PWA on a routine basis. In an effort to better understand current practice in the United Kingdom, Brumfitt [14] used a questionnaire to obtain information from 173 SLPs regarding common practices related to the importance of psychosocial factors (defined as changes in psychological state and social engagement in response to aphasia) in the management of aphasia. The data obtained suggested that practicing SLPs believed strongly in the value of psychosocial aspects as an important part of the aphasia management process. Approximately $75 \%$ of participants rou- tinely requested psychosocial information from their patients. However, there was significant variability regarding how SLPs were actually including psychosocial aspects in assessment and treatment. Thus, there is evidence suggesting that there was a strong belief in the value of QoL management, but variability in how this belief translated into clinical practice.

The purpose of this research was to gather data regarding how SLPs are implementing current recommendations regarding the assessment of QoL in PWA in the United States. This research was conducted as a pilot study to provide foundational knowledge and direction for future research questions. The questions of the current study addressed four themes related to QoL assessment: attitude, training, current practice patterns, and barriers. The clinical practice questions focused on determining how SLPs are approaching QoL in the assessment process (e.g., measures used, timing during the management process, sources of information accessed, and variability related to aphasia profiles).

\section{METHODS}

\section{Participants}

Speech-language pathologists who were currently practicing in the United States and providing services to PWA were eligible for participation in this study. According to ASHA [15], approximately $38.5 \%$ of the approximately 162,400 SLPs in the United States were employed in health care settings, suggesting about 62,500 who would likely be eligible for participation. Participants were recruited directly through e-mail (sent by one of the primary investigators) and indirectly through listervs and postcards distributed at a national conference. Participants were contacted only once with the invitation to participate. The total number of eligible people who received notice of the study was unknown.

\section{Survey}

A brief, anonymous online survey was created and made accessible using the web-based survey platform Qualtrics [16]. The final version of the survey required approximately ten minutes to complete (Appendix A). The survey began with the presentation of the informed consent form and an opportunity to select or decline participation, as well as an operational definition of QoL as explained by the WHO to address potential lack of clarity and uniformity between participants. The questions that followed addressed the following areas: demographics, training, attitudes about QoL assessment, current 
patterns of clinical practice, and perceptions of barriers. The survey consisted of a variety of question types, including forced-choice (e.g., yes/no, multiple choice) and rating scales (e.g., Likert scales, slider scales). The question design was selected with the intent of varying question types to reduce fatigue and minimize a response set bias (e.g., selecting strongly agree as the response for all survey questions). In addition, multiple question types created opportunities to elicit different types of information (e.g., using a Likert scale to determine degree of agreement versus a simple agree/disagree format).

Demographic questions included the state in which the respondent practiced, years of clinical practice as a SLP, and years of clinical practice with PWA. Questions regarding training probed whether or not formal training regarding QoL assessment was received in graduate school, from a clinical fellowship mentor (with an additional response option of don't know/remember), in a continuing education opportunity, or through self-study. Attitudes about the importance of including QoL assessment in the care of PWA was probed with a scale including seven response choices ranging from not at all important to extremely important.

In the section that addressed current patterns of clinical practice, participants were given an initial question and depending on the response provided, the survey proceeded with one of two different lines of questioning. On the key question, if the respondent indicated that he or she assessed QoL using formal measures or informal measures, then questions related to current patterns of clinical practice followed. If the participant indicated that he/she did not assess QoL in PWA, the survey prompted the respondent to explain for this choice, thus bypassing questions about clinical practice and resuming with questions regarding barriers. The clinical practice questions addressed the methods of assessment utilized as well as the following assessment parameters: frequency with which various individuals were asked to provide information, frequency of assessment at various time points in the management cycle, frequency of assessment by aphasia severity, and frequency of assessment by aphasia type.

For the question addressing the methods of assessment utilized, nine choices were provided as well as a choice to select "other" and manually enter a method that was not specifically listed. Those listed included ASHA's QCL, Communicative Activities Checklist (CAC) [17], Communication Activities of Daily Living (CADL) [18], interview, observation, self-generated tool, Social Activities Checklist (SAC) [19], Social Network Analysis (SNA) [20], and the SAQOL-39. For all the frequency- based questions, the following five response choices were provided: never, rarely, sometimes, quite often, and very often. Respondents reported on the frequency with which they accessed QoL information through the following sources of information: PWA, immediate family member (e.g., spouse, child), professional caregiver (e.g., nurse, certified nursing assistant), and the respondent's professional opinion. They also reported on the frequency in which they elicited QoL information during the following stages of management: intake/ initial evaluation, throughout treatment, at time of discharge, and after discharge. Lastly, they reported the frequency with which they assessed QoL in patients with following aphasia severities: mild, moderate, severe, and profound, and the following aphasia types: Broca's, global, anomic, and Wernicke's.

All participants were presented with questions regarding perceived barriers to the assessment of QoL in PWA (regardless of whether they indicated they did or did not assess QoL). The barriers probed in the survey included: lack of training/ knowledge, lack of available resources, insufficient time, lack of funding/reimbursement issues, language intervention taking precedence, difficulty assessing some PWA, and the issue of whether or not this is the responsibility of the SLP. For each potential barrier listed, the respondents were able to indicate the degree to which they identified this item as a barrier to QoL assessment with the following response choices: strongly disagree, disagree, neither agree nor disagree, agree, strongly agree. In addition, respondents were given the opportunity to list any other barriers they perceived in clinical practice.

The survey link was posted on the university website and the website link was distributed through e-mail and listervs. Informed consent was obtained at the start of the survey, and survey questions immediately followed. Prior to launch, the survey was piloted on three SLPs who were asked to provide feedback regarding clarity, appropriateness, and ease-of-use. These professionals reported the survey was clear, appropriate, and easy to maneuver. As a result, no changes were made to the survey prior to its official launch.

\section{Ethics, consent, and permissions}

The study was approved by the Western Carolina University Institutional Review Board in accordance with the protection of human subjects. Informed consent was obtained from all participants prior to presentation of survey questions. In the informed consent, consent to publish data was obtained from participants. 


\section{Data analyses}

The data obtained through the survey consisted of categorical data that was quantified and reported as frequency counts and percentages. Participants were not required to provide responses on every questions as some questions might not have been applicable to certain participant or may have been questions they did not want to answer. As a result, missing data occurred for some questions. Due to the limited responses for several of the choices on the Likert scale, statistical analyses were not completed at this time.

\section{RESULTS}

\section{Demographics}

Seventy-one individuals provided consent to participate in the study, but only 62 went on to complete more than just the demographic information section. The mean number of years in clinical practice was 15.28 years $(\mathrm{SD}=12.48)$ with a range of 1 year to 50 years, while the mean number of years in clinical practice specific to $\mathrm{PWA}$ was 13.3 years $(\mathrm{SD}=11.26)$, ranging from 1 year to 45 years. Categorically, 49 participants reported having over five years of clinical experience, and 20 participants reported having five years or less. A similar trend was observed regarding years of experience working with PWA; 45 participants reported having over five years of experience, and 22 indicated having five years or less. Thirty-five states from across the country were represented in the sample, and two participants did not provide information regarding location. Many respondents reported working in multiple settings, and the exact counts for each setting were as follows: inpatient/ hospital/acute (37), rehabilitation/sub-acute (33), skilled nursing facility (20), long-term care facility (13), outpatient rehabilitation (12), private practice (10), home health (8), university clinic (5), and school (1). The respondents' primary community settings were suburban (35), urban (27), and rural (20).

\section{Importance of assessing quality of life}

Of the 62 participants responding to the question, 39 (63\%) believed assessment of QoL was extremely important, 19 (30.5\%) believed it was very important, three (5\%) believed it was somewhat important, and one (1.5\%) believed it was very unimportant.

\section{Assessment of quality of life}

Sixty of the 62 participants (96.7\%) responding to the initial assessment question indicated that they used informal measures to assess QoL in PWA. For the two participants that remained, one participant indicated that he/she used formal measures (1.6\%), and the other participant reported that he/ she did not assess QoL in this population (1.6\%). Because all participants that indicated the use of formal or informal assessments subsequently received the full set of survey questions pertaining to current practices, the data from these 61 participants have been combined and presented as one group. As demonstrated in Table 1, the majority of participants responding indicated that they use interview $(59,96.7 \%)$ and observation $(54,88.5 \%)$ to gather information about the QoL of PWA. In addition, some participants $(22,36 \%)$ also indicated the use of self-generated measures. The most frequently used published measure was the CADL $(8,13 \%)$. Interestingly,

Table 1. The frequency with which respondents $(n=61)$ used the following measures and methods in the assessment of QoL in PWA. Multiple responses permitted

Survey question: Please select the quality of life measures that you have used in the past (select all that apply)

\begin{tabular}{lcc}
\hline Assessment Measure or Method & Frequency & Percentage \\
\hline ASHA's quality of communication life scale (OCL) & 5 & 8 \\
Communicative activities checklist (CAC) & 2 & 3.3 \\
Communicative activities of daily living (CADL) & 8 & 13 \\
Interview & 59 & 96.7 \\
Observation & 54 & 88.5 \\
Self-generated survey or checklist & 22 & 36 \\
Social activities checklist (SAC) & 5 & 8 \\
Social network analysis (SNA) & 2 & 3.3 \\
Stroke and aphasia quality of life scale-39 (SAOOL-39) & 3 & 5 \\
Other & 6 & 10 \\
\hline
\end{tabular}


many participants indicated that that they used not only a variety of methods, but also reported using more than one during a single session. One participant reported the use of the Voice Handicap Index [21] as an other option.

Participants reported that QoL was assessed throughout the therapy process, but seldom after the client was discharged as a follow-up effort (Table 2). More specifically, 44 (73\%) of the 60 respondents indicated that QoL was assessed quite often (16) or very often (28) at the start of treatment. A similar trend was observed when asked if assessment took place during treatment (of the 60 responding: quite often [28, 46.7\%]; very often $[27,45 \%]$ ) or at the time of discharge (of the 59 responding: quite often [12, 20.3\%]; very often [38, 64.4\%]). Only 10 $(17.2 \%)$ of 58 responses indicated that assessment was completed quite often (6) or very often (4) after a client was discharged from therapy.

Data from 60 respondents indicated that information about a client's QoL was requested from the client with aphasia quite often $(15,25 \%)$ or very often $(39,65 \%)$. Other sources of information were professional opinion (of 58 responses: 21 quite often [36\%], 27 very often [46.5\%]), immediate family members (of 60 responses, 25 quite often [41.6\%\}; 25 very often [41.6\%]), and other healthcare professionals (of 57 responses, 20 quite often [35\%], 9 very often [16\%]). These data are presented in Table 2.

Results also suggested that SLPs were assessing QoL with similar patterns of frequency across severity levels and aphasia type (Table 2). Those with a moderate level of aphasia severity were administered QoL assessments most often as 57 of 61 responses (93\%) indicated that these individuals were assessed quite often (25) or very often (32). The least likely to be assessed were those with profound aphasia, with 16 (26\%) and $29(47.5 \%)$ of 61 responses indicating that assessments were given quite often or very often, respectively. The severe

Table 2. Frequency data for stages during which assessment occurs, sources of information, severity levels of PWA assessed, and types of aphasia assessed Survey question: How often do you assess quality of life at different stages of management?

\begin{tabular}{lcccccc}
\hline & Never & Rarely & Sometimes & Quite often & Very often & Total responses \\
\hline Intake, initial evaluation & 0 & 0 & 6 & 16 & 28 & 60 \\
Throughout treatment & 0 & 0 & 5 & 28 & 27 & 60 \\
At time of discharge & 0 & 4 & 5 & 12 & 38 & 59 \\
Some time after discharge, follow-up & 12 & 18 & 18 & 6 & 4 & 58 \\
\hline
\end{tabular}

Survey question: How often do you interview the following persons when assessing the client's quality of life?

\begin{tabular}{lcccccc}
\hline & Never & Rarely & Sometimes & Quite often & Very often & Total responses \\
\hline Client with aphasia & 0 & 1 & 5 & 15 & 39 & 60 \\
Immediate family member (e.g., spouse, child) & 0 & 1 & 9 & 25 & 25 & 60 \\
Professional caregiver (e.g., nurse, certified nursing assistant) & 2 & 8 & 18 & 20 & 9 & 57 \\
Your professional opinion & 2 & 0 & 8 & 21 & 27 \\
\hline
\end{tabular}

Survey question: How often do you assess quality of life at different stages of management?

\begin{tabular}{lccccccc}
\hline & Never & Rarely & Sometimes & Quite often & Very often & Total responses \\
\hline Mild aphasia & 0 & 0 & 9 & 20 & 32 & 61 & 32 \\
Moderate aphasia & 0 & 0 & 4 & 25 & 31 & 61 \\
Severe aphasia & 0 & 2 & 9 & 20 & 10 & 29 & 61 \\
Profound aphasia & 1 & 5 & 10 & 16 & \\
\hline
\end{tabular}

Survey question: How often do you assess quality of life in persons with aphasia with the following aphasia types?

\begin{tabular}{lccccccc}
\hline & Never & Rarely & Sometimes & Quite often & Very often & Total responses \\
\hline Broca's & 0 & 0 & 5 & 23 & 33 & 61 & 61 \\
Global & 2 & 4 & 8 & 21 & 26 & 33 \\
Anomic & 1 & 1 & 4 & 22 & 23 & 61 \\
Wernicke's & 0 & 0 & 10 & 23 & 28 & 61 \\
\hline
\end{tabular}


Table 3. Frequency with which SLPs agree to various barriers of quality of life management

Survey question: To what extent do you agree to the following as barriers to the assessment of quality of life in persons with aphasia?

\begin{tabular}{|c|c|c|c|c|c|c|}
\hline & $\begin{array}{l}\text { Strongly } \\
\text { disagree }\end{array}$ & Disagree & $\begin{array}{l}\text { Neither agree } \\
\text { nor disagree }\end{array}$ & Agree & $\begin{array}{l}\text { Strongly } \\
\text { agree }\end{array}$ & $\begin{array}{c}\text { Total } \\
\text { responses }\end{array}$ \\
\hline Lack of training or knowledge & 3 & 4 & 11 & 34 & 10 & 62 \\
\hline Lack of available resources & 3 & 5 & 13 & 30 & 11 & 62 \\
\hline Insufficient time & 3 & 12 & 16 & 26 & 5 & 62 \\
\hline Lack of funding/ reimbursement issues & 3 & 15 & 18 & 16 & 10 & 62 \\
\hline Direct language intervention takes precedence & 3 & 11 & 10 & 28 & 10 & 62 \\
\hline Too difficult to assess in some persons with aphasia & 7 & 12 & 7 & 30 & 6 & 62 \\
\hline This is not the responsibility of the SLP & 34 & 19 & 7 & 1 & 1 & 62 \\
\hline Other barriers & 0 & 0 & 4 & 1 & 2 & 7 \\
\hline
\end{tabular}

and profound aphasias had counts of never or rarely, which was not observed for the mild and moderate aphasias. Finally, the frequency with which QoL assessment occurred in persons with a specific type of aphasia was also fairly consistent. Of the 61 responses obtained, persons with Broca's aphasia were found to be the most common type receiving QoL assessments, with 56 (92\%) indicating quite often (23) or very often (33). The least likely group to receive QoL assessment was persons with global aphasia, with 21 (34.4\%) indicating quite often and 26 (42.6\%) indicating very often.

\section{Perceived barriers}

Sixty-two participants responded to questions regarding potential barriers to the assessment of QoL in PWA (Table 3). From the list of potential barriers provided in the survey, the responses agree or strongly agree were indicated for lack of training/knowledge (44,71\%), lack of available resources (41, $66 \%)$, lower priority compared to direct language intervention $(38,61 \%)$, difficulty assessing this population $(36,58 \%)$, insufficient time $(31,50 \%)$, and lack of funding/reimbursement issues $(26,42 \%)$. Many clinicians responded neutrally to these barriers (i.e., neither agree nor disagree); however, 53 respondents $(85.5 \%)$ disagreed or strongly disagreed with the suggestion that assessment of QoL was not the responsibility of the SLP.

\section{Training received}

Of the 62 participants responding to these questions, the vast majority indicated that they received formal training on QoL issues in graduate school $(56,90.3 \%)$. Thirty-one (50\%) participants reported that they had participated in continuing education on the topic, and 35 (56.4\%) indicated completion of independent self-study on the topic. Although 14 could not remember, $23(37.1 \%)$ of the participants indicated that QoL was addressed by their supervisor during their clinical fellowship year.

\section{DISCUSSION}

The purpose of this study was to gain a better understanding of SLPs' application of practice recommendations regarding the assessment QoL in PWA in the United States. Data included information regarding SLPs' perceptions of the importance of QoL assessment in PWA, how SLPs are currently conducting these assessments, how SLPs learned about assessing QoL in PWA, and SLPs' perceptions of barriers in the assessment process. By understanding these variables, suggestions could be provided and future research could be conducted regarding how and where ASHA recommendations are being implemented and what can be done to improve implementation.

Almost all of the respondents reported that they had received QoL training in graduate school, and nearly half reported completion of continuing education and independent study on the issue. However, lack of training/knowledge was the number one barrier that respondents identified (agreed or strongly agreed) as a hindrance to their ability to assess QoL over any of the other choices provided on the survey. Possible reasons for this discrepancy are that training may be focused more on the concept of QoL and the importance of addressing it rather than specific diagnostic strategies or methods by which SLPs accomplish this.

Another interesting finding pertains to the tools used. Respondents reported that interview and observation were the 2 most commonly employed methods of assessing QoL in PWA. This is not surprising as both of these dynamic assessment methods can provide valuable information while allowing 
flexibility to adapt to the client's strengths and needs. However, these were often used in isolation, without other more standardized measures. There are limitations to using these methods alone, such as the lack of consistency and reliability in assessments, clinicians, and interpretations. If used correctly, published measures (e.g., ASHA QCL, SAQOL-39) inherently allow for consistency and reliability within and across administrations. The question that logically follows is: Why are clinicians primarily using informal assessment methods in isolation? This could be related to insufficient training, but other possibilities must be considered. For example, materials may not be available to clinicians and funds to acquire them may be limited or absent; current data support this as $66 \%$ of respondents agreed and or strongly agreed that availability of resources was a perceived barrier. Another possibility is that clinicians may be unfamiliar with the assessments that are available. Before specific recommendations can be made as to how to best overcome this, it is important to further explore the reasons underlying this trend.

It was also interesting to learn that the SLPs reported that QoL seldom is addressed after treatment has ended in the form of follow-up despite the reality that aphasia is a chronic disorder with life-long implications and life-long therapeutic needs. In 2011, ASHA published the National Outcomes Measurement System (NOMS) [22] reports for 2006-2010, including data specific to adults receiving care following a stroke. Considering the outpatient data in particular, some interesting practice patterns emerged. When time at the facility was completed, $88.2 \%$ of patients were discharged home, but only $26.4 \%$ of patients with stroke were recommended to receive continued speech-language therapy. This brings to light the concern of the SLP's limited involvement in the long-term recovery process despite a continued need. Kirkevold [23] interviewed nine stroke survivors asking them to identify the aspects of recovery that seemed most challenging at different periods post-onset. It was reported that after being discharged home from rehabilitation, one significant challenge was adjusting to their new reality of living with long-term disability. As reported by Ch'ng and colleagues [24], "acceptance of life changes, engagement in new roles and activities and the presence of social support appear to be key factors in post-stroke adjustment" (p. 1136). These reports and previously discussed data regarding rates of depression in persons living with chronic aphasia suggest that some form of QoL and psychosocial management is necessary in the long-term for best QoL outcomes.
Finally, for reasons not yet known, QoL of persons with more severe aphasia or global aphasia is not being assessed as regularly as those with less severe presentations of aphasia. Communicating with those with more severe aphasia is challenging for clinicians and communication partners alike, and this finding may reflect that difficulty. However, it could also be the result of assessment design issues and training to communicate with this population. To accommodate those with severe aphasia, it is appropriate to use various communication strategies to ensure the client understands the question asked and has a reliable modality through which to respond. There are assessments, such as the Assessment for Living with Aphasia (ALA) [25], which incorporate pictographic supports for this purpose. The role of proxies in this process is also important; it has been reported that persons familiar with and regularly involved in the life and care of the PWA can serve as a proxy and can complete assessments, such as the SAQOL39 , on behalf of the PWA [26].

\section{Limitations}

While our sample of SLPs from geographically diverse areas in the US does provide informative groundwork for a larger study, the number of participants who participated may not serve as a representative sample of the larger population of SLPs working with PWA. In addition, there are several factors that may have inadvertently influenced the resulting data, and revisions to the survey are necessary to continue or expand upon this line of research. It is ideal that the survey include additional questions, such as the estimated percentage of PWA comprising the clinicians' caseloads. In the list of QoL assessments choices provided on the survey, additional measures should be added, such as the ALA and the Quality of Life Questionnaire for Aphasics (QLQA) [8]. Additionally, abbreviations/acronyms for all listed measures should be provided as many SLPs refer to assessments tools in this manner rather than by the full title.

\section{Future directions}

The data obtained in this study provide guidance towards future research on this topic as well as improvements to the current study. For example, the finding that QoL assessment is commonly addressed in academic instruction and continuing education opportunities coupled with the finding that training and knowledge are a perceived as barriers begs several questions. First, how is QoL instruction/information being presented? Secondly, is there a preferred method of instruction 
that facilitates QoL recommendations being put into practice? This is an important topic to explore prior to making recommendations regarding additional and/or perhaps even different training to improve QoL assessment practices in PWA.

Another direction of research is to explore the use of interview and observation in the informal assessment process given the high rate with which interview and observation methods are used, particularly in isolation. For formal measures, the current data suggests use of the CADL more so than other measures of QoL. Future research could investigate why SLPs select one particular measure over another (e.g., availability, policy, cost, content, or reliability/validity?). Clinician preferences may influence how measures are used and even how they should be designed in the future to increase the likelihood of utilization.

Finally, it is of great concern that there is a group of people with severe aphasia who may not be considered for QoL assessment simply due to the severity of the aphasia. This issue warrants further exploration to understand what variables are contributing to this, such as the SLP's skill level to communicate with this population or adaptability of assessment tools for this population.

\section{CONCLUSIONS}

In order to provide the best possible rehabilitation, it is critical that decisions be guided by reliable and valid assessments. The aphasia literature provides SLPs with numerous empirically researched, peer-reviewed studies that are available to clinicians to support best practice in the assessment of QoL in PWA. Perhaps these findings will increase awareness among practitioners regarding the current practice and encourage them to take action to improve their own clinical practice. Several peer-reviewed studies and published assessment tools have been discussed in this paper, and this may encourage SLPs to seek out those citations. Further, these findings highlight the need for additional research to better understand where, how, and why current practice patterns are occurring so that a plan can be established to improve practice and thus outcomes. To ensure quality care and best outcome, the issue of QoL assessment must be at the forefront of discussion and research.

\section{REFERENCES}

1. World Health Organization. What quality of life? World Health Fo- rum. 1996;17(4):354-356.

2. Cruice M, Hill R, Worrall L, Hickson L. Conceptualising quality of life for older people with aphasia. Aphasiology. 2010;24(3):327347.

3. Cruice M, Worrall L, Hickson L, Murison R. Finding a focus for quality of life with aphasia: social and emotional health, and psychological well-being. Aphasiology. 2003;17(4):333-353.

4. Hilari K, Byng S. Healthy-related quality of life in people with severe aphasia. International Journal of Language \& Communication Disorders. 2009;44(2):193-205.

5. Lam JM, Wodchis WP. The relationship of 60 disease diagnoses and 15 conditions to preference-based health-related quality of life in ontario hospital-based long-term care residents. Medical Care. 2010;48(4):380-387

6. Ross K, Wertz R. Quality of life with and without aphasia. Aphasiology. 2003;17:355-364.

7. Hilari K, Needle JJ, Harrison KL. What are the important factors in health-related quality of life for people with aphasia? a systematic review. Archives of Physical Medicine and Rehabilitation. 2012;93 Suppl 1:86-95.

8. Spaccavento S, Craca A, Del Prete M, Falcone R, Colucci A, Di Palma A, et al. Quality of life measurement and outcome in aphasia. Neuropsychiatric Disease and Treatment. 2014;10:27-37.

9. Worrall L, Hickson L. Communication disability in aging: from prevention to intervention. 2003.

10. American Speech-Language Hearing Association. Preferred practice patterns for the profession of speech-language pathology. Preferred Practice Patterns. 2004. Available from: www.asha.org/ policy.

11. Ross K. Assessing quality of life with aphasia: an annotated bibliography. Perspectives on Neurophysiology and Neurogenic Speech and Language Disorders. 2005;15:15-18

12. Paul D, Frattali CM, Holland ML, Thompson C, Caperton C, Slater S. Quality of communication life scale. ASHA. 2004.

13. Hilari K, Byng S, Lamping DL, Smith SC. Stroke and aphasia quality of life scale-39 (saqol-39): evaluation of acceptability, reliability, and validity. Stroke. 2003;34:1944-1950.

14. Brumfitt S. Psychosocial aspects of aphasia: speech and language therapists' views on professional practice. Disability \& Rehabilitation. 2006; 28(8):523-524.

15. American Speech-Language Hearing Association. Asha summary membership and affiliation counts, year-end 2016. ASHA Website. 2017. Available from: www.asha.org

16. Qualtrics. Qualtrics survey software (computer software). Qualtrics Version 39660. 2013.

17. Cruice M. Communicative activities checklist. Communication Disability in Aging: From Prevention to Intervention. 2001.

18. Holland AL, Frattali CM, Fromm D. Communication Activities of Daily Living. 1999.

19. Cruice M. Social activities checklist. Communication Disability in Aging: From Prevention to Intervention. 2001.

20. Antonucci TC, Akiyami H. Social networks in adult life and a preliminary examination of the convoy model. Journal of Gerontology. 1987;42(5):519-527. 
21. Jacobson BJ, Johnson A, Grywalski C, Silbergleit A, Jacobson G, Benninger MS, et al. The voice handicap index (vhi): development and validation. American Journal of Speech-Language Pathology. 1997;6(3):66-69.

22. American Speech-Language Hearing Association. National outcomes measurement system: adults in healthcare. Outpatient National Data Report 2011.2011.

23. Kirkevold M. The unfolding illness trajectory of stroke. Disability and Rehabilitation: An International, Multidisciplinary Journal. 2002;24(17):887-898.

24. Ch'ng AM, French D, McLean N. Coping with the challenges of re- covery from stroke: long term perspectives of stroke support group members. Journal of Health Psychology. 2008;13(8):11361146.

25. Simmons-Mackie N, Kagan A, Victor JC, Carling-Rowland A, Mok A, Hoch JS, et al. The assessment for living with aphasia: reliability and construct validity. International Journal of Speech-Language Pathology. 2014;16(1):82-94.

26. Hilari K, Owen S, Farrelly SJ. Proxy and self-report agreement on the stroke and aphasia quality of life scale-39. Journal of Neurology, Neurosurgery, and Psychiatry. 2007;78(10):1072-1075. 


\section{Appendix A.}

Clinical Training: For the questions that follow, please select the most appropriate answer regarding your clinical training for managing quality of life in persons with aphasia.

1. Did you receive formal training in a graduate program?

Yes

No

2. Did your CFY supervisor address quality of life in his or her clinical practice?

Yes

No

Don't know/remember

3. Have you completed continuing education courses on this topic?

Yes

No

4. Have you completed any self-study or reviewed available literature on this topic?

Yes

No

Importance: The following questions address your opinions regarding the importance of quality of life issues in patients with aphasia.

1. In your opinion, how important is it that speech-language pathologists assess quality of life in patients with aphasia?

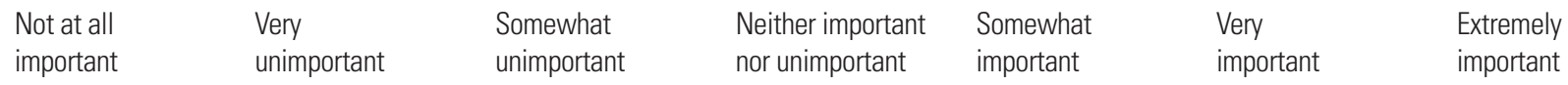

\section{Assessment}

1. How do you assess quality of life in persons with aphasia?

$\begin{array}{lll}\text { Formal measures } & \text { Informal measures } & \text { I do not assess } \\ \text { (e.g., scale, survey) } & \text { (e.g., interview) } & \text { quality of life }\end{array}$

2. Please select the quality of life measures that you have used in the past: (select all that apply)

- ASHA's quality of communication life scale

- Communicative activities checklist

- Communicative activities of daily living

- Interview

- Observation

- Self-generated survey or checklist

- Social activities checklist

- Social network analysis

- Stroke and aphasia quality of life scale-39

$\circ$ Other:

3. How often do you interview the following persons when assessing client's quality of life?

a. Client with aphasia

Never Rarely Sometimes Quite often Very often

b. Immediate family member (e.g., spouse, child)

Never Rarely Sometimes Quite often Very often

c. Professional caregiver (e.g., nurse, certified nursing assistant)

Never Rarely Sometimes Quite often Very often

d. Your professional opinion

Never Rarely Sometimes Quite often Very often

4. How often do you assess quality of life at different stages of management?

a. Intake, initial evaluation

Never Rarely Sometimes Quite often Very often

2. What is the primary reason that you do not assess quality of life in persons with aphasia?

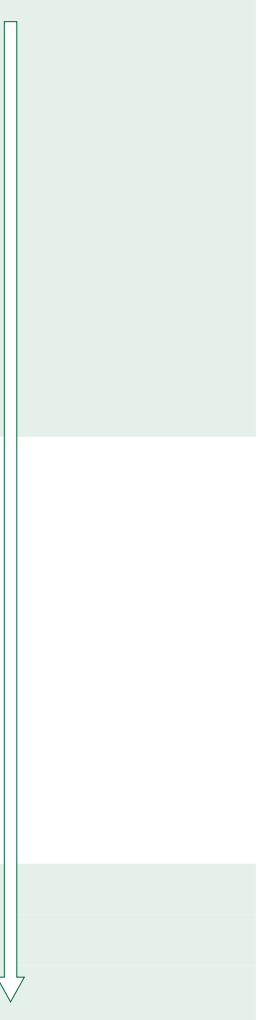

(Continued to the next page) 
Appendix A. Continued

\begin{tabular}{|c|c|c|c|c|}
\hline \multicolumn{5}{|c|}{ b. Throughout treatment } \\
\hline Never & Rarely & Sometimes & Quite often & Very often \\
\hline \multicolumn{5}{|c|}{ c. At time of discharge } \\
\hline Never & Rarely & Sometimes & Quite often & Very often \\
\hline \multicolumn{5}{|c|}{ d. Some time after discharge, follow-up } \\
\hline Never & Rarely & Sometimes & Quite often & Very often \\
\hline
\end{tabular}

5. How often do you assess quality of life in persons with ap hasia of varying severity levels?

a. Mild aphasia

$\begin{array}{llll}\text { Never } & \text { Rarely } & \text { Sometimes } & \text { Quite often }\end{array}$

b. Moderate aphasia

Never Rarely Sometimes Quite often Very often

c. Severe aphasia

Never Rarely Sometimes Quite often Very often

d. Profound aphasia

Never Rarely Sometimes Quite often Very often

6. How often do you assess quality of life in persons with aphasia with the following aphasia types?
a. Broca's
Never
Rarely
Sometimes
Quite often
Very often
b. Global
Never
Rarely
Sometimes
Quite often
Very often
c. Anomic
Never
Rarely
Sometimes
Quite often
Very often
d. Wernicke's

Never Rarely Sometimes Quite often Very often

7. To what extent do you agree to the following as barriers to the assessment of quality of life in persons with aphasia?

\begin{tabular}{|c|c|c|c|c|c|}
\hline a. Lack of training or knowledge & Strongly disagree & Disagree & $\begin{array}{l}\text { Neither agree nor } \\
\text { disagree }\end{array}$ & Agree & Strongly agree \\
\hline b. Lack of available resources & Strongly disagree & Disagree & $\begin{array}{l}\text { Neither agree nor } \\
\text { disagree }\end{array}$ & Agree & Strongly agree \\
\hline c. Insufficient time & Strongly disagree & Disagree & $\begin{array}{l}\text { Neither agree nor } \\
\text { disagree }\end{array}$ & Agree & Strongly agree \\
\hline d. Lack of funding/ reimbursement issues & Strongly disagree & Disagree & $\begin{array}{l}\text { Neither agree nor } \\
\text { disagree }\end{array}$ & Agree & Strongly agree \\
\hline $\begin{array}{l}\text { e. Direct language intervention takes } \\
\text { precedence }\end{array}$ & Strongly disagree & Disagree & $\begin{array}{l}\text { Neither agree nor } \\
\text { disagree }\end{array}$ & Agree & Strongly agree \\
\hline $\begin{array}{l}\text { f. Too difficult to assess in some persons } \\
\text { with aphasia }\end{array}$ & Strongly disagree & Disagree & $\begin{array}{l}\text { Neither agree nor } \\
\text { disagree }\end{array}$ & Agree & Strongly agree \\
\hline g. This is not the responsibility of the SLP & Strongly disagree & Disagree & $\begin{array}{l}\text { Neither agree nor } \\
\text { disagree }\end{array}$ & Agree & Strongly agree \\
\hline h. Other barriers: & & & & & \\
\hline
\end{tabular}

